\title{
Estudios radiográficos determinantes para el conocimiento constructivo de tres esculturas policromadas realizados por el CCRBC de Castilla y León
}

\author{
Consuelo Valverde Larrosa, Cristina Gómez González, Juan Carlos Martín García, Pilar Vidal \\ Meler, Milagros Burón Álvarez
}

Resumen: En este artículo se presentan los resultados del análisis radiográfico de tres esculturas de bulto redondo construidas con materiales distintos. Por medio del estudio de RX se determina el modo constructivo de las piezas y el estado de conservación de los materiales utilizados. Este tipo de estudios desvelan los secretos de las obras y son fundamentales para la investigación en profundidad sobre nuestro patrimonio cultural.

Palabras claves: Análisis radiográfico; Estudio multiespectral; Escultura de papelón; Caña de maíz; Busto relicario; Estudio multidisciplinar; Metodología de trabajo.

Determinantes estudos radiográficos para o conhecimento construtivo de três esculturas policromadas, realizados pelo CCRBC de Castilla e León

Resumo: Neste artigo, apresentam-se os resultados da análise radiográfica de três esculturas de vulto redondo, construídas com materiais distintos. Através deste estudo de RX, determina-se o modo construtivo das peças e o estado de conservação dos materiais constituintes. Este tipo de estudo revela os segredos das obras e é fundamental para uma profunda investigação do nosso patrimonio cultural.

Palavras-chave: Análises radiográficas; Estudo multiespectral; Escultura de papelão; Caule do milho; Busto relicário; Estudo multidisciplinar; Metodologia de trabalho.

Determinant radiographic studies for the constructive knowledge of three polychrome sculptures, carried out by CCRBC of Castilla y León

\begin{abstract}
In this article, we present the results of the radiographic analysis of three sculptures in round built with different materials. Through this study of RX, the constructive process and the conservation status of the constituent materials are determined. This kind of study reveals the secrets of the artworks and is essential for a thorough investigation of our cultural heritage.
\end{abstract}

Keywords: Radiographic analysis; Multispectral study; Cardboard sculpture; Corn stalk; Bust reliquary; Multidisciplinary study; Research methodology.

\section{Introducción:}

Entre las técnicas de estudios físicos habitualmente utilizadas en el Centro de Conservación y Restauración de Bienes Culturales de Castilla y León (C.C.R.B.C. de C. y L.) para el estudio de los Bienes Culturales están la reflectografía infrarroja, la radiografía y la fluorescencia ultravioleta. Estas técnicas, junto con la fotografía digital en el rango visible, aportan registros permanentes para su estudio, que debemos interpretar correctamente con el fin de conocer de forma integral los bienes culturales y poder planificar una adecuada intervención sobre ellos. 
Este conjunto de estudios multiespectral sin toma de muestras, se lleva a cabo en el C.C.R.B.C. de C. y L. como método de trabajo utilizando la radiación electromagnética de diversas longitudes de onda.

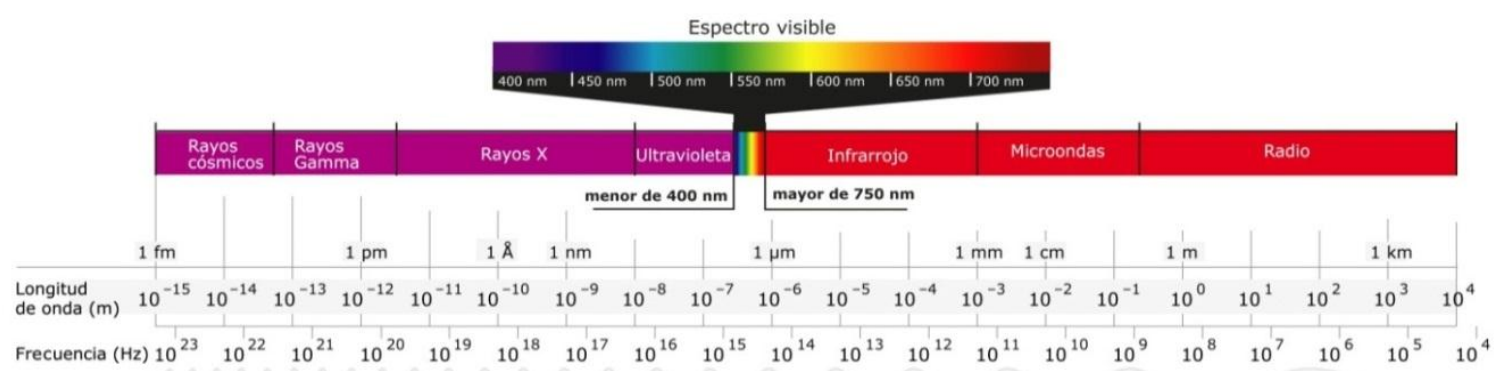

Figura 1. Gráfico que especifica las longitudes de onda y su frecuencia, correspondientes a cada espectro

En este artículo nos centraremos en los estudios con rayos X. Este tipo de radiación es capaz de atravesar cuerpos sólidos y dejar una impronta permanente sobre un soporte fotosensible de lo que encuentra a su paso, por lo que nos aporta valiosa información en cuanto al estado de conservación de policromías, reutilización de lienzos, añadidos de piezas, estructuras internas, ahuecados, elementos "extraños" etc. Los estudios físicos se complementan con los estudios químicos de caracterización de materiales, tanto de la policromía como del soporte, mediante micromuestras tomadas de diversas zonas de las obras.

El estudio integral de las obras en el C.C.R.B.C. de C. y L. es multidisciplinar, implicándose los diversos departamentos con el fin de conseguir un objetivo común. En este artículo se pretende evidenciar cómo el estudio radiográfico es fundamental para la comprensión integral de las obras que vamos a intervenir. Mostraremos un resumen de los resultados de los estudios radiográficos que se llevaron a cabo en el C.C.R.B.C. de C. y L. sobre tres esculturas de distinta naturaleza, y cómo estos estudios fueron fundamentales para desvelar cómo fueron construidas las obras. Consideramos que el momento de la restauración es único para realizar este tipo de investigaciones, ya que se separan de su ubicación habitual por un tiempo, pudiendo manipularlas libremente y en un lugar adecuado para su estudio.

Hemos elegido tres esculturas de bulto redondo con características materiales muy distintas entre sí, en los que la radiografía ha sido imprescindible para su comprensión estructural y constructiva. Hablaremos del "Ecce Homo" perteneciente a la iglesia parroquial de Santiago el Real en Medina del Campo (Valladolid), catalogado como de la segunda mitad del siglo XVII y realizado con papel encolado. De principios del siglo XVII, realizado con papel amate, cañas y pasta de maíz, es el "Cristo Crucificado" de la Colegiata de San Pedro de Lerma (Burgos). Por último, encuadrado en el siglo $\mathrm{XVI}$ y realizado en madera de roble, se analiza un busto relicario perteneciente a la iglesia parroquial de Joarilla de las Matas (León). 


\section{Proceso radiográfico}

EI C.C.R.B.C. de C. y L. cuenta con un equipo generador de RX, GEIT SEIFER, modelo ERESCO 160 MRF3.1, de $160 \mathrm{kV}$ y 3,7mA de tensión e intensidad máxima. Empleamos película médica de columna vertebral cuya longitud máxima es $120 \times 30 \mathrm{~cm}$., una vez impresionada la película se revela automáticamente con una procesadora AGFA "Curix 60" durante 3 minutos a 34 grados. Siempre que sea posible, se realiza la radiografía en un solo disparo, optimizando la seguridad radiológica del personal y la calidad de la imagen que se obtiene. Una vez reveladas, cada placa se pasa a formato digital y se unen con programas de tratamiento de imagen para obtener una imagen de RX completa.

Habitualmente se trabaja en un búnker en el C.C.R.B.C. de C. y L., pero como el equipo es portátil podemos emplearlo en campo si así lo requiere la obra, como es el caso de uno de los ejemplos que se muestran en el artículo.

\section{Estudio Radiográfico}

\section{Ecce Homo}

Se trata de un busto perteneciente a la iglesia parroquial de Santiago el Real de Medina del Campo (Valladolid), datado en la segunda mitad del siglo XVII, cuyas dimensiones son $60 \mathrm{~cm} \times 55 \mathrm{~cm} \times 30 \mathrm{~cm}$. y tiene un peso total de $3,340 \mathrm{Kg}$. Está constituido fundamentalmente por papel, tela encolada y policromada, ojos de cristal, pestañas y pelo natural, cordón con hilo metálico, soga policromada para la corona y peana de madera (Martín 2012).

Nuestro interés residía en averiguar cómo estaba construido el busto para poder realizar un tratamiento integral de restauración, corrigiendo una deformidad estructural que se traducía en la excesiva inclinación del busto hacia atrás. Necesitábamos saber si estaba realizado completamente con papel y si la tela encolada a modo de clámide se había colocado por encima, o sólo tenía papel en las zonas visibles y el resto estaba realizado con telas encoladas. También nos interesaba averiguar si existía una estructura interna sobre la que descansara la imagen o era completamente hueco. La imagen se radiografió con todos los atributos colocados ya que nos suscitaba gran interés su heterogeneidad material.

\section{Características de la imagen radiográfica}

Se realizó la radiografía, tanto en vista frontal como lateral, uniendo dos placas dobles en disposición vertical para cada vista. En ambos casos se obtuvo la imagen completa con un solo disparo, aunque cambiando ligeramente las condiciones. Para la vista frontal se disparó a $45 \mathrm{kV}, 6 \mathrm{~mA}$, a 2 metros de distancia y durante 1,10 minutos. Para hacer el perfil se cambió la tensión a 55kV manteniendo los otros parámetros.

Según consta en el análisis estratigráfico realizado el Laboratorio de Química del C.C.R.B.C. de C. y L. (Barrera 2012), la preparación tiene distinta composición en la clámide que en las carnaciones. La clámide está compuesta por sulfato cálcico y cola animal, mientras que la carnación presenta abundante blanco de plomo. La presencia de este material da un aspecto característico en la placa radiográfica. 


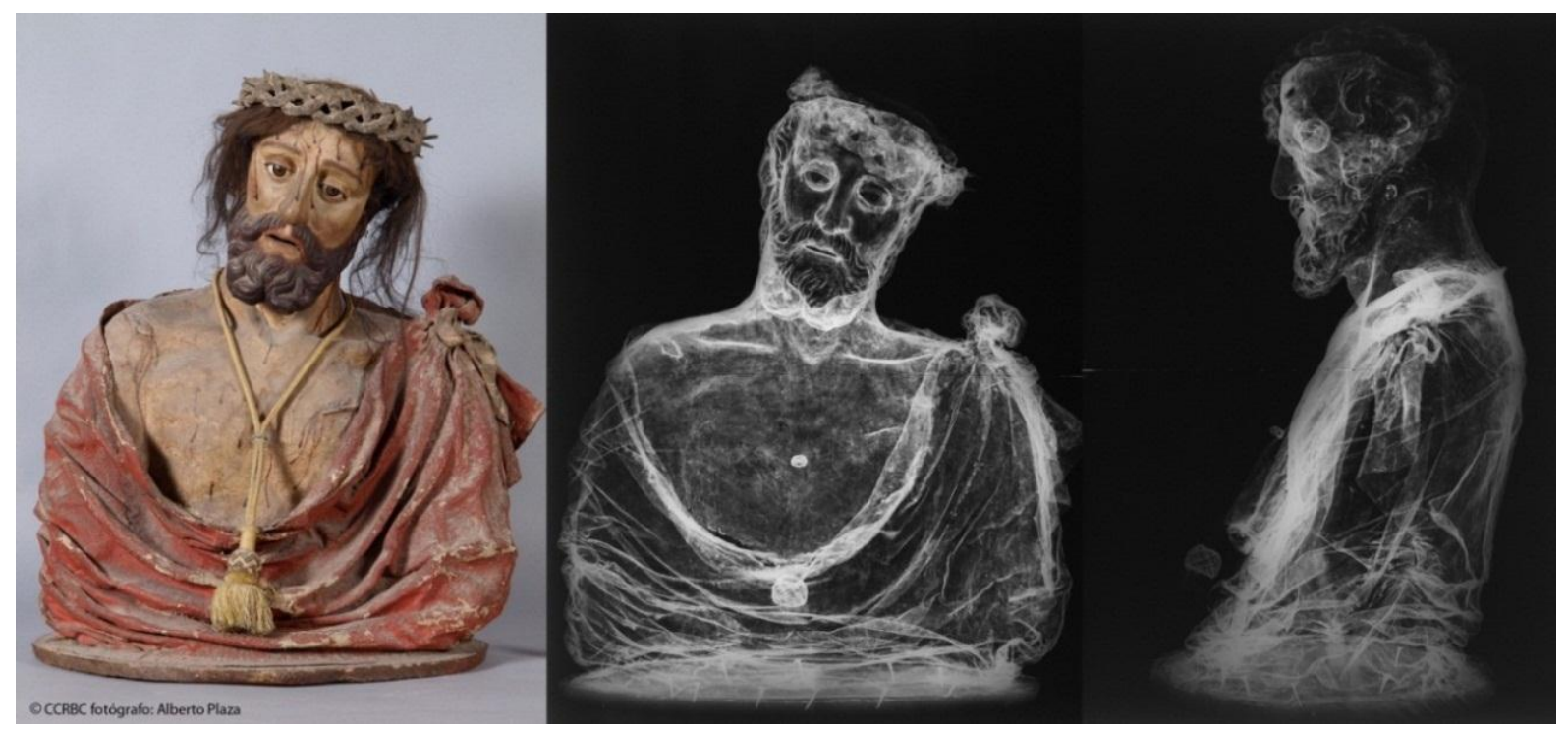

Figura 2. Ecce Homo en el espectro visible y RX (de frente y de perfil)

\section{Características del soporte}

Según la imagen radiográfica obtenida podemos ver en el lateral del brazo izquierdo una línea de mayor absorción radiográfica que llega hasta la base (las referencias de zonas se refieren siempre según vemos la imagen). Además la superficie que está cubierta por la clámide absorbe mucha más radiación que el resto, por lo que estábamos en condiciones de asegurar que se trataría de un busto realizado completamente con papel encolado al que se ha superpuesto la clámide de tela encolada.

En el interior solamente se observa una zona rectangular más radiopaca, que nos indicaría la presencia de una pieza colocada en oblicuo desde la base, a unos $13 \mathrm{~cm}$. del extremo derecho, y continúa en oblicuo hasta que apoya en el extremo derecho. El listón mide $42 \mathrm{~cm}$. de longitud y tiene $1,5 \mathrm{~cm}$. de anchura (medidas tomadas sobre la placa radiográfica). Se ve tanto en el frontal como en el perfil. Podríamos identificarlo como una pieza de refuerzo, que se colocó antes de ponerle la base para hacer presión y mantener el volumen de la pieza en ese punto.

El hecho de no tener ninguna estructura interna nos lleva a pensar que quizá lo construyeran a partir de un molde previo, que les sirviera de apoyo para pegar el papel encolado y que, tras el secado, pudieran desmoldar; obteniendo de este modo el volumen y la forma deseada. Posteriormente, sobre el busto obtenido, colocarían las telas dándole el acabado buscado.

En cuanto a elementos metálicos, encontramos un clavo actual en el nudo de la clámide, usado como refuerzo de unión al busto, y un total de 12 clavos de forja en la base, que nos revelan el modo de unir el busto a la base: los clavos están introducidos de arriba hacia abajo, quedando las cabezas casi a la vista. La altura de la base es de $1 \mathrm{~cm}$., y podemos distinguir la dirección de la veta en la placa radiográfica. Como hemos dicho anteriormente, la clámide está realizada con telas encoladas y policromadas, lo que deja en nuestra placa radiográfica la huella tanto de la trama como de la urdimbre, huellas que se pueden observar de modo más evidente en las zonas de lagunas cromáticas. 
Los ojos son de cristal y de dos tipos; el izquierdo es un globo completo de vidrio que se muestra como una zona de mayor absorción radiográfica con forma esférica que podemos ver muy claramente en el perfil. En la vista frontal se distingue sutilmente la niña de la pupila, pero es indicativo de la diferencia material en cuanto a la absorción de radiación. En este mismo ojo una línea muy blanca describe un círculo que, al analizarlo en la vista de perfil, corresponde a una pieza cilíndrica de $0,8 \mathrm{~cm}$. (medida tomada sobre la placa radiográfica) que aparece a continuación del globo ocular. Probablemente sea una pieza de madera que sirviera para colocarlo en su posición correcta.

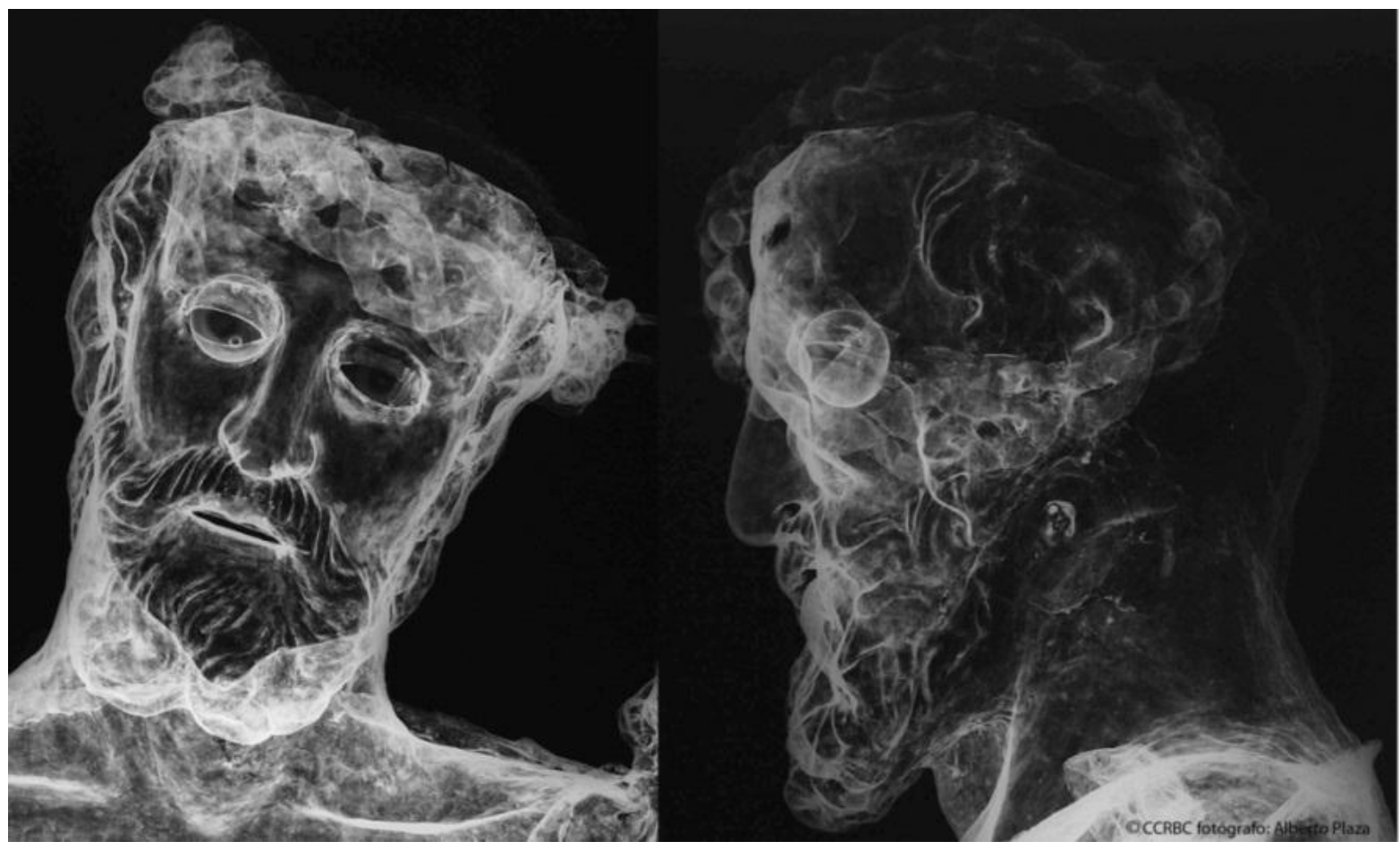

Figura 3. Detalle de la cabeza, vista frontal y perfil

En el caso del ojo derecho (según vemos la imagen) no es un globo completo, nos da una línea más radiopaca que define el ovalo del ojo. También se observa una sutil diferencia en cuanto a la absorción de radiación de la niña y de la pupila. Refiriéndonos al estado de conservación, en este caso aparece una línea muy blanca vertical rodeada de un área bastante absorbente a la radiación que correspondería a acumulación material, probablemente responde a una reparación del soporte, quizá a una rotura del ojo que se haya reforzado con algún material.

También se registran en la placa los atributos, aparecen dos óvalos, uno más grande que el otro, con un entramado de líneas en forma de nido. Corresponden a los dos nudos del cordón, compuestos por hilos metálicos que son muy radiopacos. El resto del cordón no nos deja señal, lo que nos indica que solamente tiene hilos metálicos en los nudos. La corona está realizada con soga policromada que impresiona muy sutilmente nuestra placa radiográfica.

\section{Cristo Crucificado de Lerma}

El Cristo, datado a principios del siglo XVII, está ubicado a unos diez metros de altura, presidiendo el retablo mayor de la Colegiata de San Pedro de Lerma (Burgos), que alcanza una altura total apro- 
ximada de quince metros. Pudimos acceder a él gracias al proyecto de restauración del retablo, promovido por la Fundación de Patrimonio de Castilla y León, en colaboración con la parroquia de San Pedro de Lerma, y cuya dirección facultativa corría a cargo del Centro de Conservación y Restauración de Bienes Culturales de Castilla y León.

Mide en centímetros $230 \times 243 \times 55$ y su peso es de 14 kilos (sin la cruz). Su gran ligereza se debe al material que lo constituye: papel amate, madera de frondosa del género Erithrina, en concreto de colorín, cañas y pasta de maíz, identificado por el Laboratorio de Química del C.C.R.B.C. de C. y L. (Barrera 2012), aspecto que, sin duda, facilitó mucho las cosas para emplazarlo en el lugar que ocupa.

\section{Características de la imagen radiográfica}

El estudio radiográfico se realizó en la propia iglesia, bajando el Cristo del retablo. Fue fundamental mantener un área de seguridad radiológica controlada, ya que la película se impresionó en la propia iglesia. El entorno nos era propicio pues se encuentra situada en una zona elevada de la ciudad y rodeada de naturaleza en la cara norte. Además el gran espesor de los muros nos servía para contener la radiación.

Se pudieron desmontar los brazos que se encontraban desencolados, lo que facilitó la manipulación, pudiendo radiografiar tanto el frente como el perfil. Según el estudio estratigráfico realizado por el Laboratorio de Química del C.C.R.B.C. de C. y L. (Barrera 2012), la película pictórica está compuesta por blanco de plomo, lo que opone mayor resistencia a la radiación y refleja un tono blanquecino en toda la superficie de la placa radiográfica.

Se realizaron un total de cuatro disparos en las condiciones que se muestran en la siguiente tabla.

Figura 4. Características de radiografiado

\begin{tabular}{|l|l|c|c|c|c|}
\hline Fecha & \multicolumn{1}{|c|}{ Zona } & $\mathbf{k V}$. & $\mathbf{m A}$. & Tiempo (Minutos) & $\begin{array}{c}\text { Distancia } \\
\text { (Metros) }\end{array}$ \\
\hline $19 / 04 / 2011$ & Frente cuerpo completo & 100 & 5 & 1,4 & 4 \\
\hline $19 / 04 / 2011$ & Perfil cuerpo completo & 110 & 5 & 1,4 & 4 \\
\hline $19 / 04 / 2011$ & Perfil Cabeza & 110 & 5 & 1,4 & 4 \\
\hline $19 / 04 / 2011$ & Brazos frente & 100 & 5 & 1,4 & 4 \\
\hline
\end{tabular}

Gracias a la colaboración del Instituto del Patrimonio Cultural de España (IPCE) utilizamos película industrial tipo II ASTM/D-7 de Agfa en formato de rollo, revelándola con procesadora Structurix NDT1 durante 8 minutos a 30 grados. Después se escanearon las placas con un escáner de transmisión ARRAY 2905 HD. Se utilizaron un total de 13 metros de película que hubo que cortar a medida para radiografiar tanto el frente como el perfil del Cristo y el frente de los brazos.

El estudio radiográfico fue decisivo para comprender la técnica de construcción de esta majestuosa escultura novohispana. 


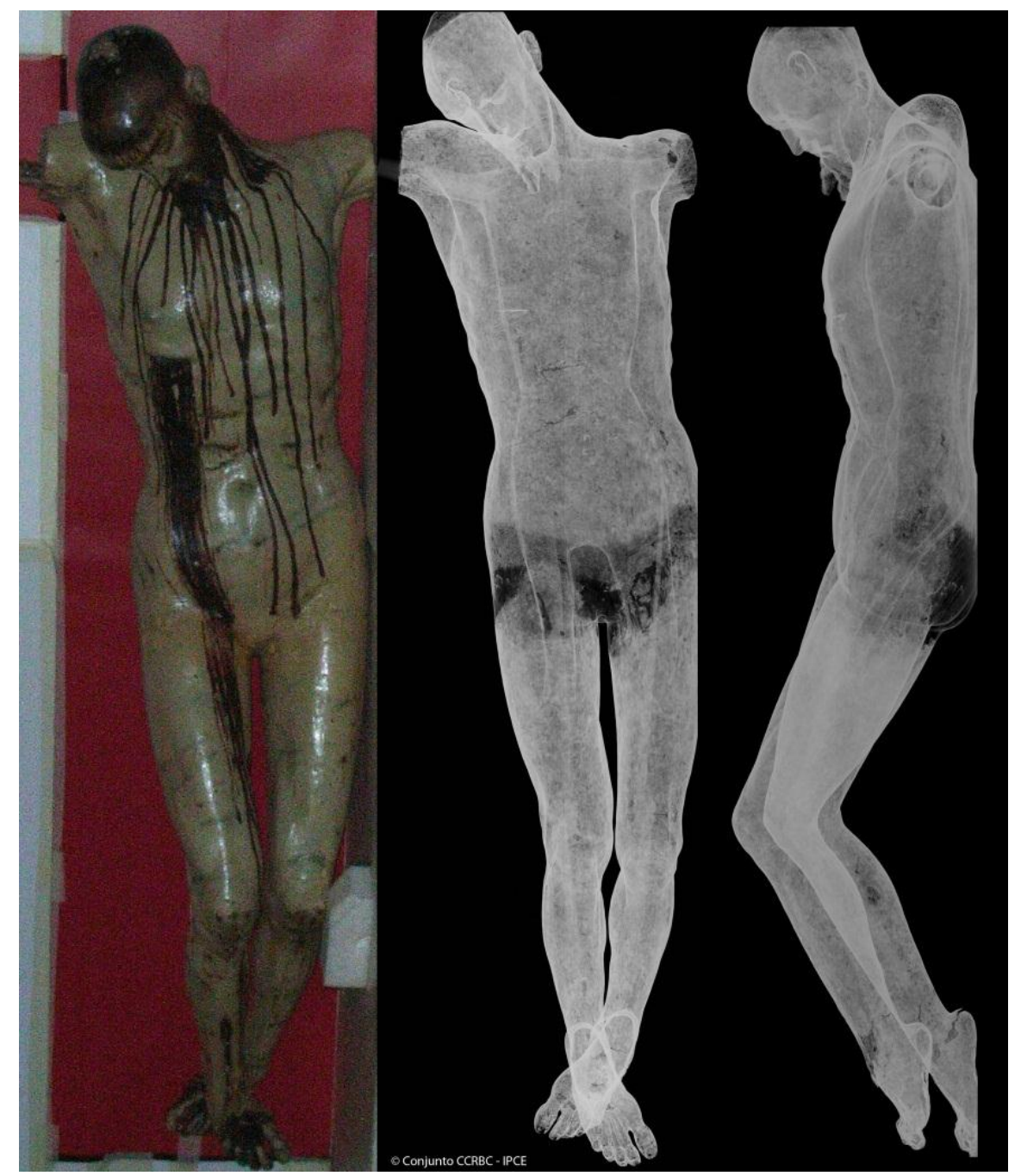

Figura 5. Cristo Crucificado en el espectro visible y RX (de frente y de perfil)

\section{Características del soporte}

Tanto en la vista frontal como en la lateral aparece una línea de mayor absorción radiográfica que discurre en paralelo al perímetro del Cristo, desde la cabeza hasta la mitad de ambos muslos. Es decir, tiene un hueco interior a modo de horma, sin ser una estructura interna propiamente dicha, ya que se trata más bien de un "maniquí" hueco con un contorno similar al exterior, sobre el que terminan de dar volumen y forma. De este modo podían construir esculturas de gran formato y peso ligero.

Si medimos sobre la radiografía en vista frontal, los resultados que obtenemos son los siguientes: la horma interna, a la altura de los hombros tiene $23,61 \mathrm{~cm}$, en la cintura $16,66 \mathrm{~cm}$ y en la cadera $23,96 \mathrm{~cm}$. El grosor de cañas desde la horma hasta el exterior es de aproximadamente $6,47 \mathrm{~cm}$. 


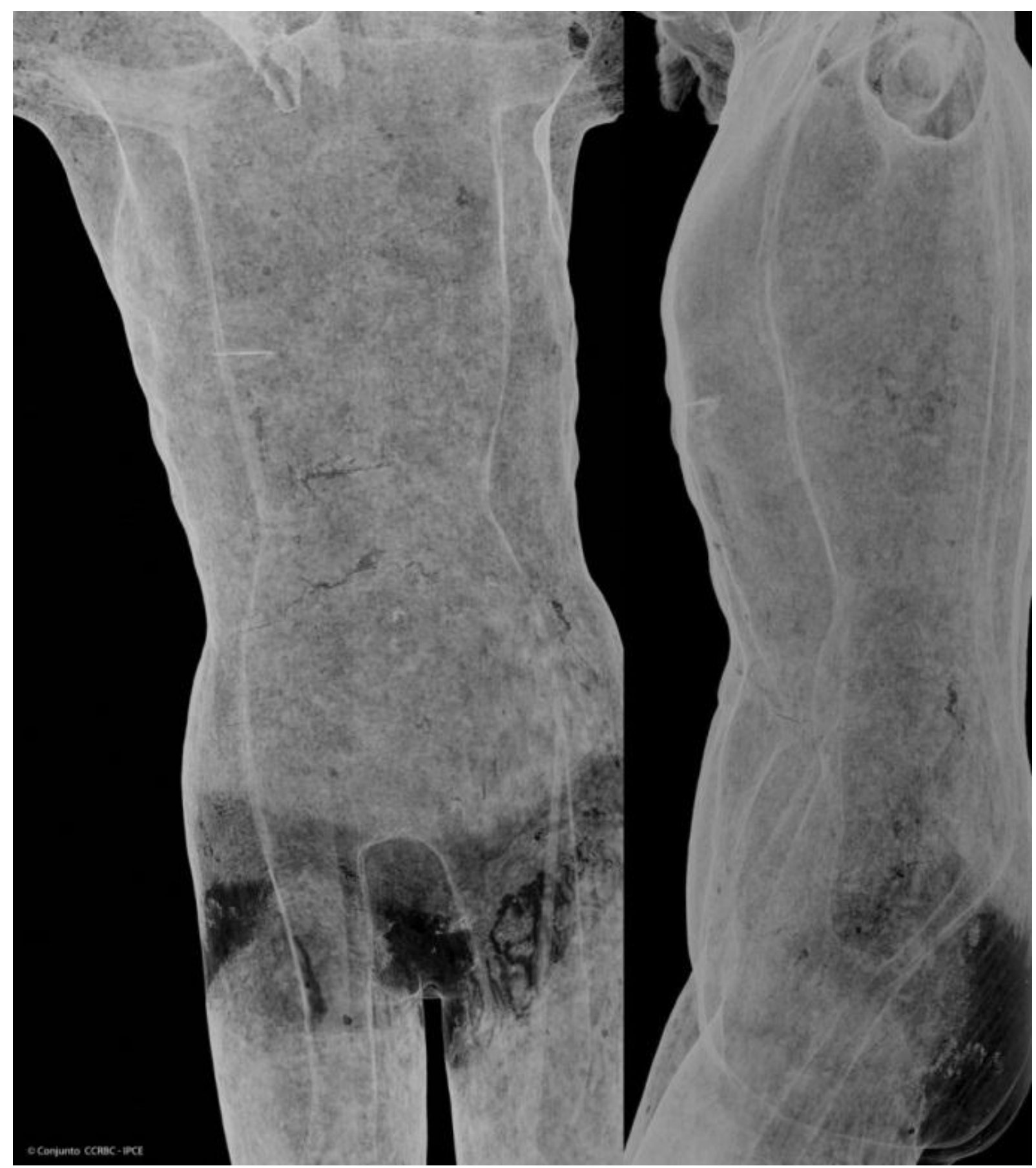

Figura 6. Detalle del tronco en vista de perfil y frontal. Horma interior.

Para construir las piernas sí utilizan unos listones de madera, que entran en el hueco de la horma; en el caso de la pierna derecha (según se mira la imagen) llega casi a la cadera; fuera de la horma, sobre el listón se maciza con cañas de maíz consiguiendo el volumen final deseado. El tipo de madera probablemente sea el mismo que se identificó en los pies: madera de frondosa del género Erithrina. 


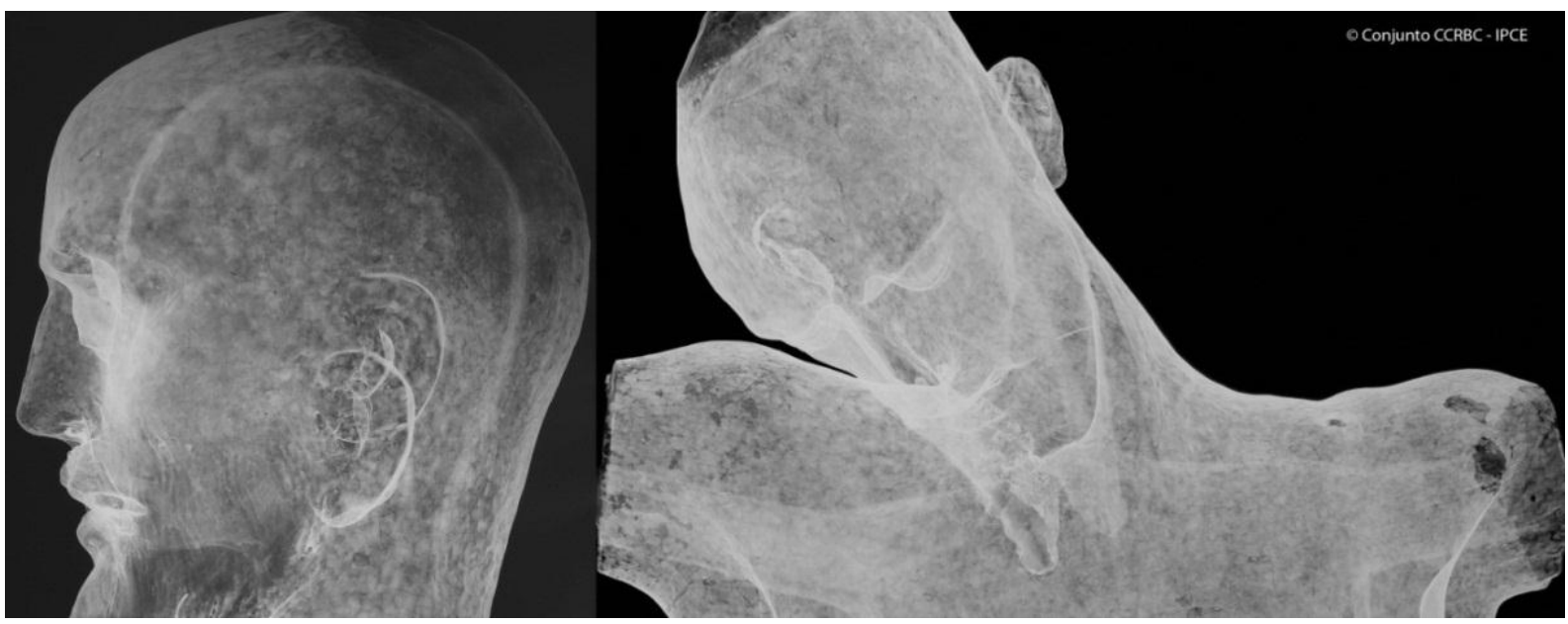

Figura 7. Detalle de la cabeza en vista de perfil y frontal. Horma interior.

La respuesta radiográfica en la cabeza es igual que en el tronco, hay un globo hueco y sobre él se disponen las cañas de maíz. Sin embargo los brazos responden a la misma tecnología constructiva que las piernas, pues no parten de una horma hueca, sino que se trazan macizos con las cañas de maíz.

Mediante la bibliografía consultada y el estudio radiográfico comprendimos el proceso de construcción de la obra. Posiblemente se parte de un modelo de figura humana esculpida en barro. Sobre ese modelo se aplican hojas de papel amate impregnadas en cola, cuando está cubierto por completo y seco el papel amate, lo desmoldan cortándolo por el lateral obteniendo así dos mitades, que unirán bien con papel amate o cosiéndolo. Esta técnica se practica de la misma manera tanto en el cuerpo, como en la cabeza. Para unir la cabeza con el tronco usaban un vástago (podemos ver una señal más clara en la placa). El molde del cuerpo llega hasta la mitad del muslo y a partir de aquí se ayudan de un listón de madera ligera por cada pierna a la que irán dando la forma y el volumen con las cañas de maíz.

En colaboración con el servicio de Restauración de la Diputación Foral de Álava, se realizó un estudio con videoendoscopio de $350 \mathrm{~cm}$ de lanza con visión frontal y perpendicular de la marca Olympus Opticla CO.LTD, modelo ILK-C. El objetivo utilizado fue el de la referencia AT60S-IV6C5. El acceso a la horma interna se realizó por los cilindros huecos que ensamblan los hombros con los brazos. Pudimos identificar la textura del papel amate así como las costuras de unión de las piezas desmoldadas. Conseguimos llegar hasta la mitad del muslo, donde empezaban a macizarse las piernas, pudiendo fotografiar las cañas de maíz y la pieza de madera que sirve de guía.

Para conseguir el volumen final de la imagen, el Cristo de Lerma está terminado mediante cañas de maíz adheridas a la horma interna, y sobre éstas se aplica pasta de maíz que unifica la superficie y da la posibilidad de tallar las zonas que les interese. Por último, lleva la capa de preparación y sobre ésta la película pictórica. Las extremidades (brazos y piernas), carecen de horma interna, son macizos, construidos a base de cañas de maíz, pasta y el acabado de preparación con policromía.

El estudio endoscópico también permitió documentar en el interior de la horma unos dibujos a modo de pictogramas tributarios. A todos estos estudios físicos y químicos hemos de añadir el histórico y estilístico aportado por Pablo F. Amador de la Universidad Nacional Autónoma de México, que encuadra la imagen de Lerma dentro de la producción del taller denominado de "Los grandes Cristos" (Amador 2012). 


\section{Busto Relicario de Joarilla de la Matas}

En el año 2011 ingresan en el C.C.R.B.C. de C. y L. dos bustos relicarios pertenecientes a la iglesia parroquial de Joarilla de las Matas (León) para proceder a su restauración. A ambos se les realizó un estudio radiográfico con resultados muy similares, por lo que nos centraremos en uno de ellos para detallar los datos obtenidos, en concreto del busto con número de registro 415.2.

Mide en centímetros $52 \times 41 \times 20$ y pesa $4,678 \mathrm{~kg}$. Está realizado en madera de roble dorada y policromada (Vidal 2012) y data del siglo XVI. En la parte superior de la cabeza tiene una tapa triangular que da acceso a un hueco poco profundo y forrado con tela. Nos interesaba conocer la zona interna de la imagen, cómo está construida y si contenían más reliquias en su interior.

\section{Características de la imagen radiográfica}

Se realizó el estudio radiográfico obteniendo la imagen en vista frontal y de perfil, en ambos casos en un solo disparo. Fueron necesarias dos placas dobles de columna vertebral, dispuestas en vertical para obtener la vista frontal, mientras que para la vista lateral se empleó una sola placa doble. Para la toma frontal se disparó a $80 \mathrm{kV}, 5 \mathrm{~mA}$, a una distancia de 2,10 metros, durante 0,50 minutos y para el perfil se aumentó la tensión a $90 \mathrm{kV}$ manteniendo el resto de los parámetros.

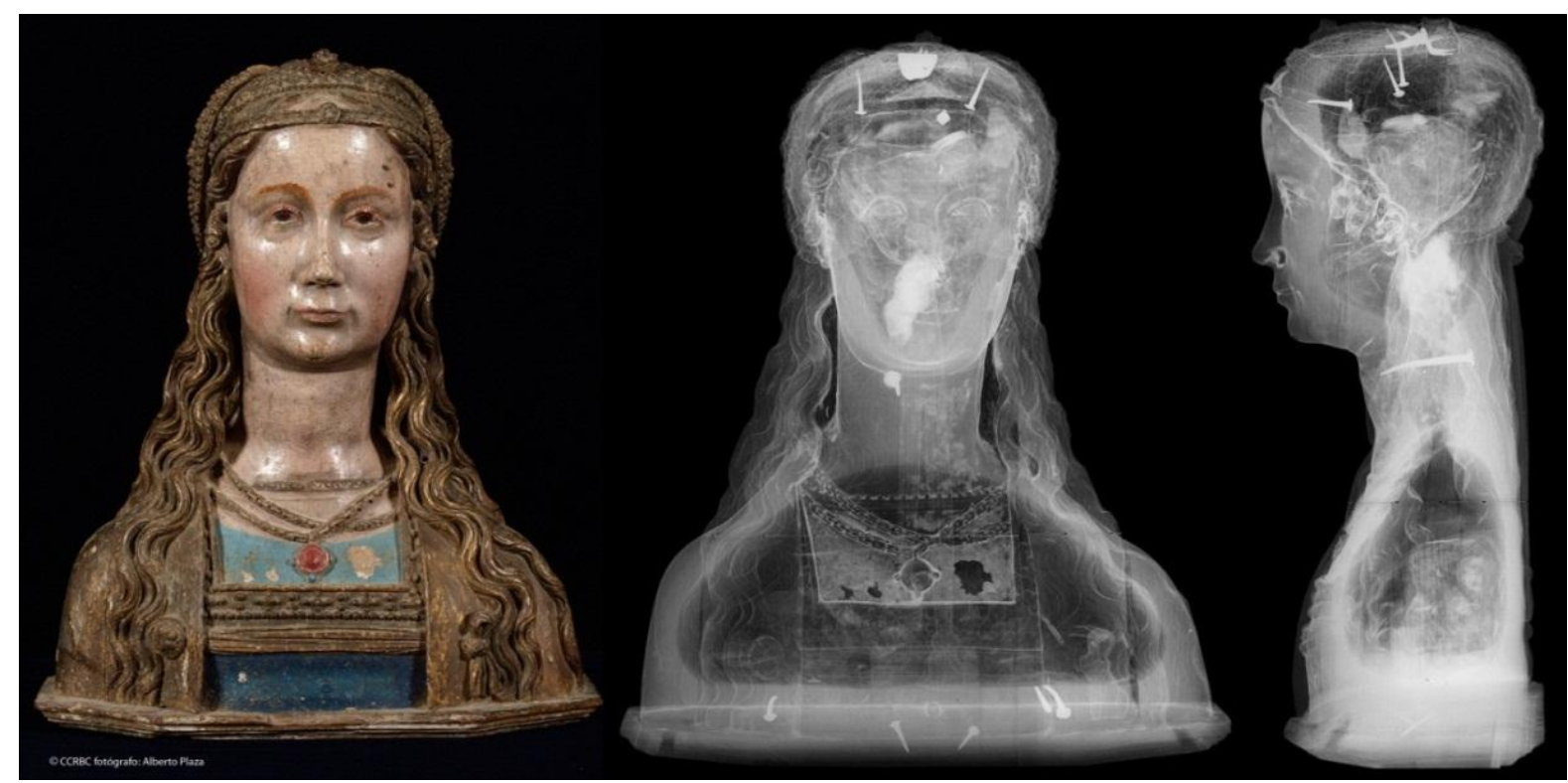

Figura 8. Busto Relicario en el espectro visible y RX (de frente y de perfil)

\section{Características del soporte}

En la vista radiográfica del perfil observamos dos líneas verticales más absorbentes que corresponden a los ensambles de unión de las tres piezas que constituyen el volumen completo del busto. Las tres piezas tienen un grosor muy similar, unos $7 \mathrm{~cm}$ (medidos sobre la placa radiográfica). Las dos principales constituyen el reverso y la zona central del busto, y la tercera pieza construiría el volumen correspondiente al pecho y al rostro. En la vista frontal observamos la línea de unión de 
dos piezas más que correspondería a los hombros, la peana y la espiga interior de unión entre la pena y el busto. Por tanto, tenemos un total de siete piezas principales, de madera de roble, que constituyen el volumen completo del busto. Los ensambles están realizados a unión viva y llevan unas espigas de madera de refuerzo para asegurar la unión. En la placa radiográfica no hemos podido localizar estas espigas, pero han quedado marcadas en la policromía debido a los movimientos naturales de la madera. Se distinguen fácilmente en la unión de los hombros o en el pecho.

Lo más llamativo de la imagen radiográfica fue la presencia de dos zonas menos absorbentes a la radiación, situadas en la cabeza y en el pecho, que hemos de identificar como huecos en el interior de la escultura (dejan una señal oscura en la placa). Por la descripción del ensamblado de piezas y la bibliografía consultada al respecto, podemos afirmar que el ahuecado se hacía en las piezas principales, antes de ser ensambladas.

Dentro de las zonas de vaciado tenemos áreas de mayor absorción radiográfica, que evidencian la presencia de reliquias en el interior. Era habitual envolver los restos óseos con telas "preciosas", sedas doradas, adornadas con hilos metálicos, tachuelas metálicas, o mediante entorchados, conformando un "saquito" que albergaría reliquias. Así se refleja en la cabeza del busto relicario, donde encontramos hilos y adornos metálicos evidenciados en la placa radiográfica como líneas muy luminosas. También se observan áreas más difuminadas pero muy absorbentes, que podríamos relacionar con "saquitos" de reliquias o acumulación de telas. En la vista frontal distinguimos una mancha blanquecina muy definida a la altura de la frente, que en este caso correspondería a una reliquia sin envolver en telas, por lo que se distingue la forma de manera más nítida.

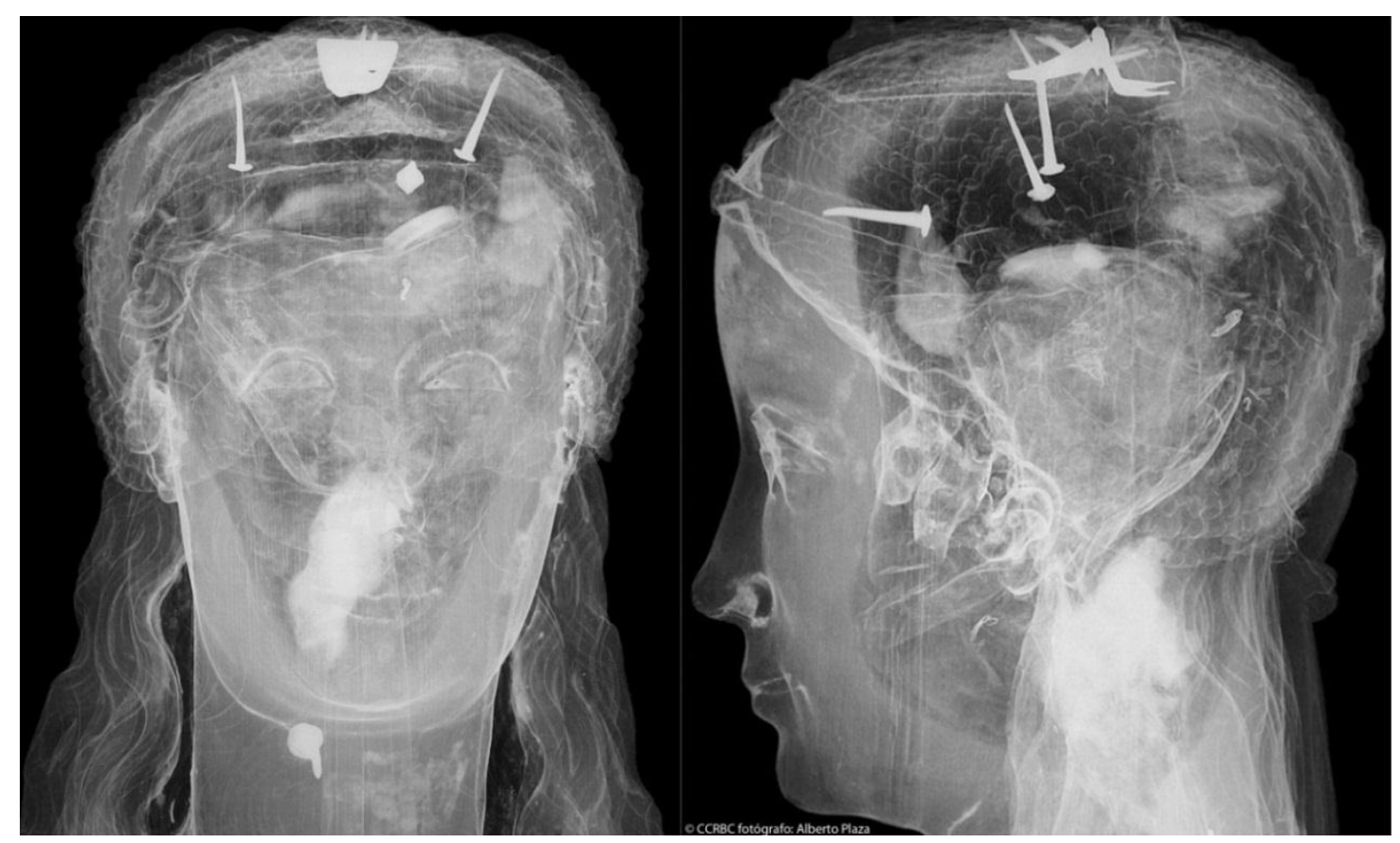

Figura 9. Vaciado interior de la cabeza, vista frente y perfil. Detalle vaciado cabeza. Elementos metálicos, restos óseos y telas

La zona del pecho también está ahuecada del mismo modo que la cabeza y hay señales radiográficas que indicarían la presencia de reliquias en su interior. En este caso la señal es más confusa, ya que tenemos muchos elementos decorativos que interfieren en la lectura de la imagen. Del análisis de la imagen radiográfica se deduce que las reliquias eran introducidas en el interior al hacer el 
ahuecado y antes de ensamblarlas, quedando ocultas y protegidas por el busto que las alberga. El ahuecado de la cabeza llegaría hasta la barbilla y el del pecho desde el comienzo del cuello hasta la base.

Por último haremos referencia a los elementos metálicos. Solamente debería aparecer la bisagra de la tapa junto con cuatro clavos de anclaje. Pero, además, se localizan tres clavos en la cabeza, uno en el cuello y cinco más en la base, que podemos relacionar con reparaciones realizadas a lo largo de la historia, seguramente para anclar piezas que tenían algo de holgura.

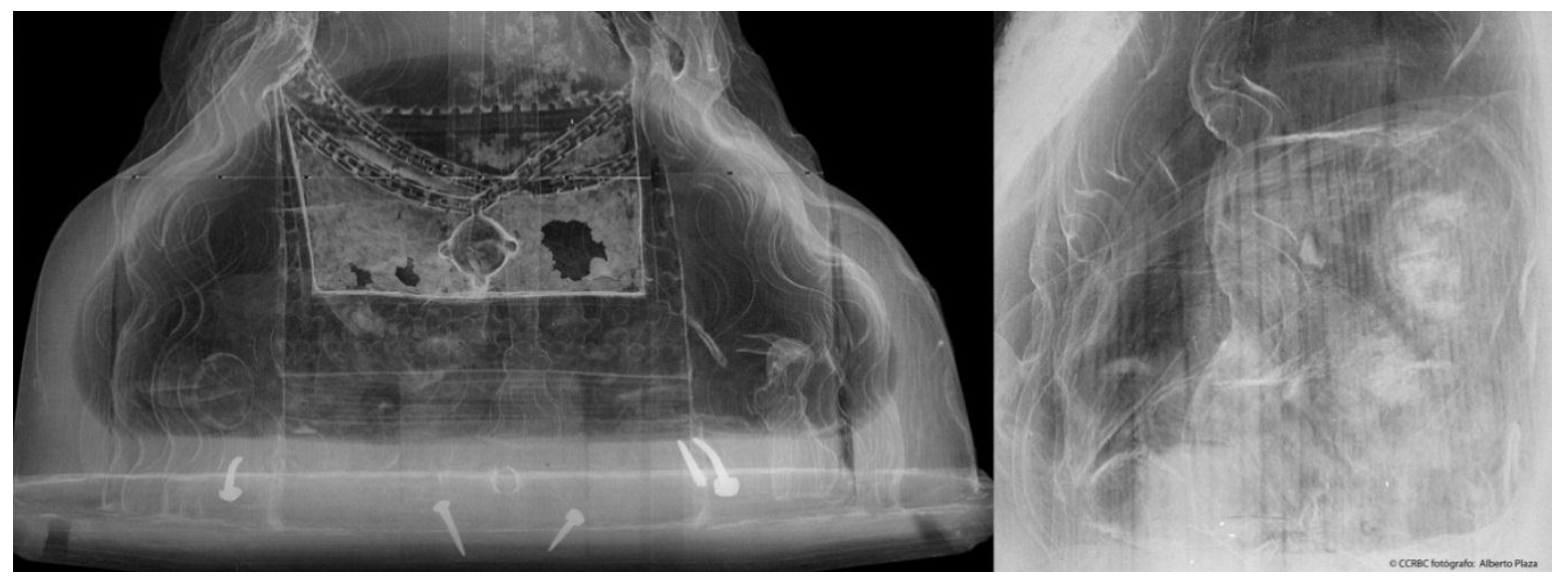

Figura 10. Vaciado interior del pecho, vista frontal y de perfil. Telas y restos óseos.

\section{Conclusiones}

Los estudios previos a las intervenciones de Restauración son fundamentales para conocer y profundizar en el proceso creativo de los maestros escultores en distintas épocas y talleres, dependiendo del material que emplearan y de la finalidad y uso de la escultura. Sólo a través de un planteamiento multidisciplinar podemos llegar a comprender las obras en su conjunto.

En los casos que se han mostrado en este artículo el estudio radiográfico ha sido imprescindible para desvelar los misterios constructivos que se esconden bajo la policromía. Si no se hubiera realizado nos quedaríamos en la superficie, en la mera acción mecánica de levantar suciedad o barnices oxidados, de reparar grietas o reintegrar cromáticamente. Sin embargo, con el estudio radiográfico hemos podido evidenciar y comprender la técnica constructiva de las tres esculturas.

El estudio en profundidad de estos bienes implica la necesidad de utilizar los avances tecnológicos a nuestro favor para acercarnos a sus orígenes, su historia, y averiguar en qué estado han llegado hasta nuestros días. Este tipo de pruebas deben incorporarse como metodología de trabajo de forma sistemática, ya que siempre aportan datos fundamentales para el conocimiento integral de nuestro patrimonio cultural y para abordar con garantías las intervenciones que se realizan sobre el mismo.

\section{Agradecimientos}

Quisieramos mostrar nuestro más profundo agradecimiento a todos quienes han colaborado de uno u otro modo en la investigación realizada: 
- Al IPCE. Sección de Estudios Físicos. Área de investigación y Formación, autores del Revelado, digitalización y tratamiento informático de las placas radiográficas del Crucificado de Lerma, especialmente al Equipo de trabajo formado por Ángeles Anaya, Tomás Antelo, Rocío Bruquetas, Miriam Bueso y Carmen Vega.

- Al Servicio de Restauración de la Diputación Foral de Álava, Soledad Rojo, Marina López, Emilio Ruiz de Arcaute y al Instituto de Investigaciones Estéticas de México,UNAM, Pablo F.Amador.

Y por supuesto a todo el equipo del C.C.R.B.C. de C. y L. Gracias a todos.

\section{Bibliografía}

AA.VV. (2010) El nacimiento de una pintura: De lo visible a lo invisible. Valencia: IVCR, Museo de Bellas Artes de Valencia.

ALBA, L. y JOVER, M. (2009). “Niños jugando a los dados de Pedro Núñez de Villavicencio. Historia de una obra a través de su radiografía", Ge-conservación, 0:47-61.

AMADOR, P.F. (2002). "Puntualizaciones sobre la imaginería "tarasca" en España. El Cristo de Telde (Canarias): Análisis y proceso de restauración, Tenerife: Universidad de La Laguna (Canarias), 157-173.

AMADOR, P.F. (2004). Traza española, ropaje indiano. Las Palmas: Universidad de Las Palmas de Gran Canaria.

BARRERA, M. (2011). "Análisis químico del crucificado". En Memoria de restauración del Cristo crucificado del retablo Mayor de la iglesia de San Pedro en Lerma (Burgos). Archivo de la Dirección General de Patrimonio Cultural de la Junta de Castilla y León.

BARRERA, M. (2012). "Análisis químicos del busto del Ecce Homo". En Memoria de restauración del busto del Ecce Homo de la iglesia de Santiago el Real en Medina del Campo (Valladolid). Archivo de la Dirección General de Patrimonio Cultural de la Junta de Castilla y León.

GABALDÓN, A., ANTELO, T., y VEGA, C. (2010). "Estudio radiográfico del soporte de obras de dos autores castellanos del siglo XV: Pedro Berruguete y Fernando Gallego". En La pintura europea sobre tablas. XV, XVI y XVII. Madrid: Ministerio de Cultura, Secretaría General Técnica, 134-138.

GABALDÓN, A., ANTELO, T., y VEGA, C. (2010). "Técnicas de imagen aplicadas al estudio de pinturas sobre tabla", En La pintura europea sobre tablas. XV, XVI y XVII. Madrid: Ministerio de Cultura, Secretaría General Técnica, 139-147.

ANTELO, T., GABALDÓN, A. y VEGA, C. (2004). "Fernando Gallego en Trujillo: Estudios Físicos", Patrimonio Cultural de España, 8: 61-73.

ANTELO, T. Y GABALDÓN, A. (2004). "Radiografía de gran formato", Patrimonio Cultural de España, 0: 202-213.

GARCÍA, R. y RUIZ, E. (2000) "Los bustos relicarios de las once mil vírgenes. Claves para su estudio", Akobe, 1: 18-21.

LABASTIDA, L. (2005) "El empleo de la videoscopia en el estudio de la imaginería ligera o de pasta de caña". Anales del instituto de investigaciones estéticas, 87: 199-216.

MARTín, J.C. (2012). Memoria de restauración del busto del Ecce Homo de la iglesia de Santiago el Real en Medina del Campo (Valladolid). Archivo de la Dirección General de Patrimonio Cultural de la Junta de Castilla y León.

VALERO, A.C. "Cristos 'tarascos'. Un ejemplo custodiado en el convento de Carmelitas descalzas de Santa Teresa de Valladolid". Valladolid, Universidad de Valladolid, 1071-1078. 
VALLEBUENO, M. (2000). "El Señor de mezquital: Un Cristo de caña del siglo XVI en Durango", Anales del Instituto de Investigaciones Estéticas, 76: 255-258.

VIDAL, P. (2012). Memoria de restauración de dos bustos relicarios de la iglesia parroquial de Joarilla de las Matas (León). Archivo de la Dirección General de Patrimonio Cultural de la Junta de Castilla y León.

VILLANUEVA, E. y AMADOR, P. "Descubrimiento de Códice mexicano en el Crucificado de Bornos, Cádiz" en Videoteca del Patrimonio Histórico Andaluz. http://www.youtube.com/watch?v=Uz62bv5nrRY [Consulta: 04/12/2009]

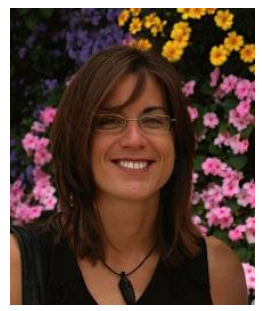

\section{Consuelo Valverde Larrosa}

consuelovalverdelarrosa@gmail.com

Consuelo Valverde Larrosa, trabaja como supervisora de la instalación radiactiva del Centro de Conservación y Restauración de Bienes Culturales de Castilla y León, especializada principalmente en el análisis radiográfico de las obras de arte. Es Licenciada en BB.AA. en la especialidad de Restauración de pintura y escultura, por la Universidad Santa Isabel de Hungría de Sevilla. Ha trabajado como restauradora para diferentes Fundaciones y empresas privadas, así como en tareas de investigación para varios museos, MNCARS, ARTIUM de Álava, y en el Museo Histórico de Écija.

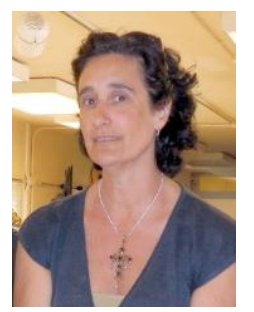

Cristina Gómez González

gomgoncr@jcyl.es

Cristina Gómez González, es Licenciada en Bellas Artes, especialidad de Restauración de pintura y escultura, por la Universidad Complutense de Madrid.

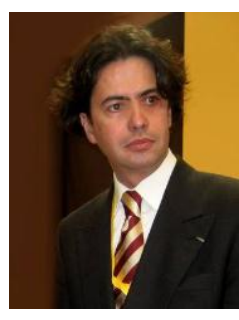

\section{Juan Carlos Martín García}

margarj@@jcyl.es

Juan Carlos Martín García es Licenciado en Bellas Artes, especialidad de Restauración de pintura y escultura, por la Universidad Complutense de Madrid. 


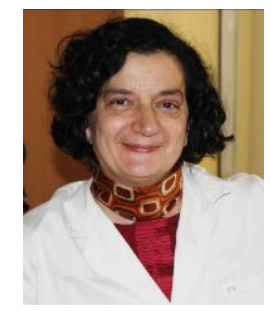

\section{Pilar Vidal Meler}

vidmelpi@jcyl.es

Pilar Vidal Meler, es Licenciada en Bellas Artes, especialidad de Restauración de pintura y escultura, por la Universidad de Barcelona.

Cristina Gómez, Juan Carlos Martín y Pilar Vidal; forman parte del Departamento de Pintura y Escultura del Centro de Conservación y Restauración de Bienes Culturales de Castilla y León. Realizan funciones de Restauración de obras de arte, Elaboración de informes y memorias finales, Redacción de proyectos de diagnosis y valoración de trabajos de restauración, Dirección técnica del desarrollo de los trabajos de restauración, Asesoramiento técnico para montajes de exposiciones, traslado de bienes culturales, proyectos museísticos.

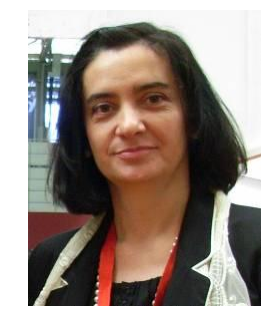

Milagros Burón Alvarez es

buralvmi@jcyl.es

Milagros Burón Alvarez es Licenciada en Filosofía y Letras Especialidades de Arqueología e Historia Antigüa. Desde el año 2001 hasta 2008 ha ejercido como Técnico Arqueólogo en la Dirección General de Patrimonio Cultural de la Consejería de Cultura y Turismo de la Junta de Castilla y León. También ha trabajado en el ámbito docente como Profesora Asociada en la Universidad de León. en el Area de Arqueología. Desde noviembre de 2008 desempeña el puesto de Directora de Centro de Conservación y Restauración de Bienes Culturales de la Junta de Castilla y León.

Artículo recibido el 30/03/2012

Artículo aceptado el 05/10/2012 